Human and Animal Health

Vol.61: e18160536, 2018

http://dx.doi org/10.1590/1678-4324-2018160536

ISSN 1678-4324 Online Edition
BRAZILIAN ARCHIVES OF

BIOLOGY AND TECHNOLOGY

AN INTERNATIONAL JOURNAL

\title{
Early and Accurate Model of Malignant Lung Nodule Detection System with Less False Positives
}

\author{
Senthilkumar Krishnamurthy ${ }^{1 *}$, Ganesh Narasimhan ${ }^{2}$, Umamaheswari Rengasamy $^{3}$ \\ ${ }^{I}$ Rajalakshmi Institute of Technology - Electronics and Communication, Kuthambakam, Chennai, Tamilnadu, India. \\ ${ }^{2}$ Saveetha University - Saveetha Engineering College, Electronics and Communication, Chennai, Tamil Nadu, India. \\ ${ }^{3}$ Velammal Engineering College - Electrical and Electronics, Velammal, Tamil Nadu, India
}

\begin{abstract}
The objective of this work is to identify the malignant lung nodules accurately and early with less false positives. 'Nodule' is the $3 \mathrm{~mm}$ to $30 \mathrm{~mm}$ diameter size tissue clusters present inside the lung parenchyma region. Segmenting such a small nodules from consecutive CT scan slices are a challenging task. In our work Auto-seed clustering based segmentation technique is used to segment all the possible nodule candidates. Efficient shape and texture features $(2 D$ and $3 D)$ were computed to eliminate the false nodule candidates. The change in centroid position of nodule candidates from consecutive slices was used as a measure to remove the vessels. The two-stage classifier is used in this work to classify the malignant and benign nodules. First stage rule-based classifier producing $100 \%$ sensitivity, but with high false positive of 12.5 per patient scan. The BPN based ANN classifier is used as the second-stage classifier which reduces a false positive to 2.26 per patient scan with a reasonable sensitivity of $88.8 \%$. The Rate of Nodule Growth (RNG) was computed in our work to measure the nodules growth between the two scans of the same patient taken at different time interval. Finally, the nodule growth predictive measure was modeled through the features such as compactness (CO), mass deficit (MD), mass excess (ME) and isotropic factor(IF). The developed model results show that the nodules which have low CO, low IF, high MD and high ME values might have the potential to grow in future.
\end{abstract}

Keywords: Lung cancer, 3-D Image Segmentation, 3-D image features, Volume growth, Lung nodule classifier.

\footnotetext{
*Author for correspondence: tkseneee@gmail.com
} 


\section{INTRODUCTION}

Worldwide lung cancer causes maximum cancer deaths than any other cancer ${ }^{1}$. Lung cancer diagnosis is also critical compared to other cancer diagnosis procedure ${ }^{2}$. The radiologist first analyzes the CT scan on the patient and suggest for one another CT scan, if any symptom of lung cancer is spotted. Physicians will not recommend for biopsy unless they found the reasonable evidence from the CT scan. This procedure of wait and watch approach makes the lung cancer diagnosis time critical. Nodule is a $3 \mathrm{~mm}$ to $30 \mathrm{~mm}$ roughly round tissue clusters exists inside the lung parenchyma region. These nodules are the primary indicator of lung cancer. Visualizing these nodules in 3D and measuring the growth of it from earlier and a later scan of the same patient makes the lung cancer diagnosis efficient. This work is concentrating on developing a mathematical model to predict the nodules which have the potential to grow in future.

Many works carried out in the field of lung nodule classifications. The computer-aided approach for detecting lung nodule consist of three fundamental steps: 1.Lung nodule candidate segmentation $^{3,4,5}, 2$.Nodule feature extraction ${ }^{6,7,8}$, and 3.Malignant nodule classification ${ }^{9,10}$. Later some work on lung nodule growth was reported in the literature. W.J.Kostis et $\underline{a l}^{11}$, developed 3-D methods for the segmentation and characterization of nodules imaged using CT. A volumetric growth characterization was formulated in this work, and this was the first such a nodule growth based system reported for clinical use. Yuanjie Zheng et al ${ }^{12}$ applied a $2 \mathrm{D}$ graphcut algorithm on the three-dimensional lung and tumor datasets. This method eliminates the possible volume variations of the nodule and estimates the tumor growth accurately. M Hasegawa et al $^{13}$ computed a volume doubling time (VDT) based on the exponential model using follow-up CT images. All the above works concentrating on measuring the nodule growth accurately, but not much on predicting the nodule growth from earlier CT scan. In this work, we are proposing a structural model to predict the nodules growing nature from earlier CT scan.

\section{MATERIALS AND METHODS}

\section{Database}

VOLCANO and LIDC are the two open source databases used for this work ${ }^{14,15}$. 34 patient cases from VOLCANO database and ten patient CT scan images from LIDC database are used in this work. For each patient in VOLCANO database, an earlier and follow-up scan CT images are available. The CT scans were taken using GE Medical and Philips CT scanners with different X-ray tube current and exposure. The time span between the scans is not specified for the VOLCANO database; however, information on nodule location is available. A total of 2646 cross sections of CT images from 44 cases were analyzed in this work. Totally 48 nodules are suspected to be malignant in this database by radiologists.

\section{Auto-Seed Clustering Based Lung Nodule Segmentation}

Segmenting a lung nodule from the lung parenchyma region is a complex task as the size, shape and the position of the nodules are differs much. Well-circumscribed, juxta-pleural, pleural tail and vascularized are the four types of nodules present in the lung parenchyma region. Segmenting all these nodules with the common algorithm is a challenging task. We already carried out some works on segmenting these nodules using threshold, region grow, and level set evolution methods ${ }^{16,17,18,19}$. In this work, we are using the concept of data clustering to segment those nodules from each slice of CT scan. Block histogram processing is carried out in this work as shown in the figure1 to choose the initial cluster seed automatically. In this research 3-D segmentation of nodules achieved by consecutive 2-D segmentation of nodule structure from each CT scan image. 

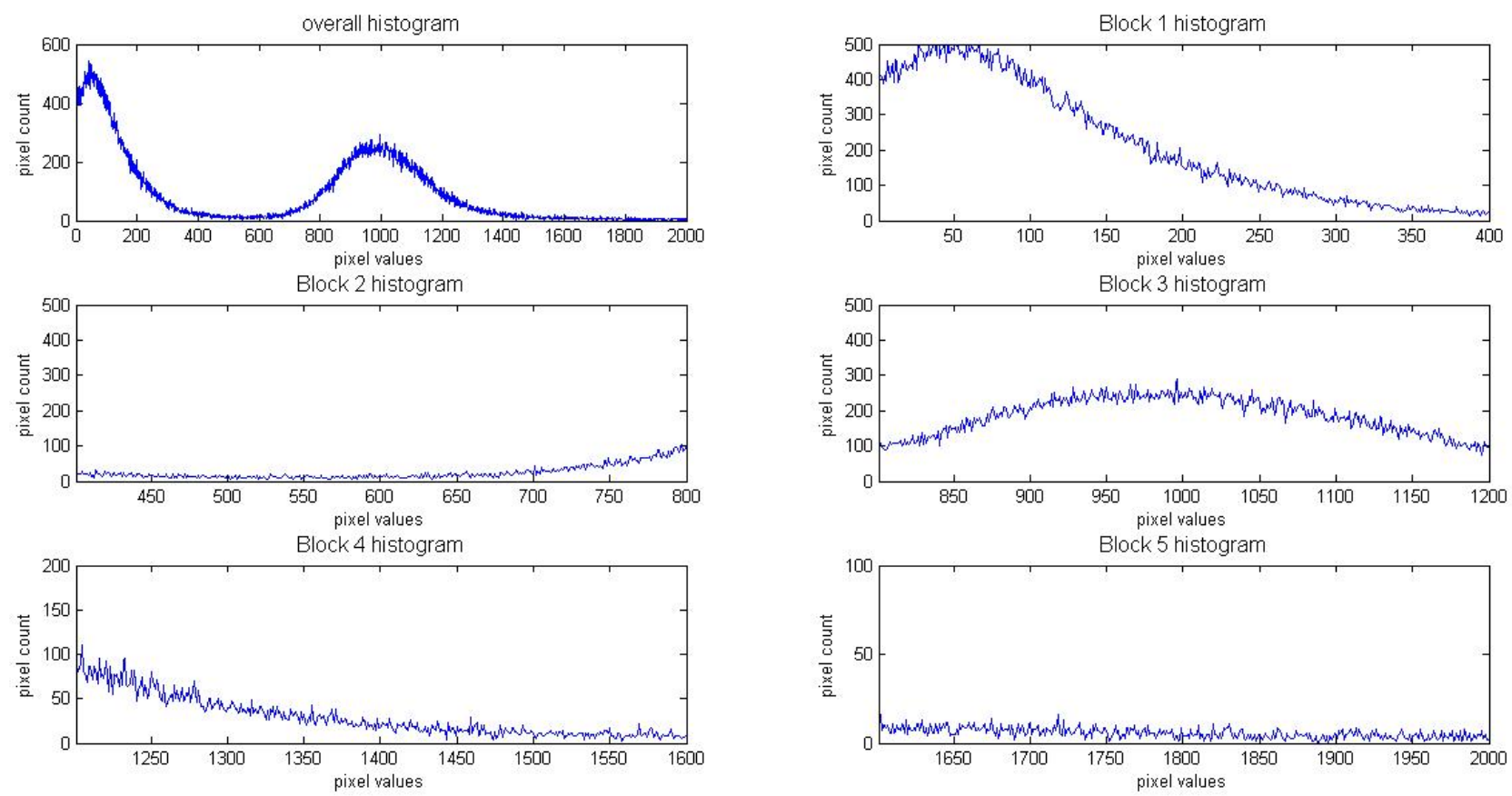

Figure 1: Block histogram to select initial seed

The proposed auto-seed clustering based segmentation algorithm steps are:

Step 1: Compute the histogram of lung CT image.

Step 2: Split the histogram into five blocks.

Step 3: Compute the pixel value corresponding to the maximum peak of each histogram block.

Step 4: Choose the mean of first two blocks maximum peak pixel position as cluster 1 seed and the mean of two blocks maximum peak pixel position as cluster 2 seed.

Step 5: Group the image pixels which have minimum distance with cluster seed1 and seed two separately without changing its spatial positions.

Step 6: Repeat from step 1 until the two consecutive cluster seeds are equal.

Step 7: Perform morphological closing and opening operation on a cluster 2 reference image to segment nodule candidates.

Step 8: Repeat the steps 1 to 7 for all the CT images slices.

Step 9: Stack the each nodule candidates segmented from each slice to form a 3D visualization. 


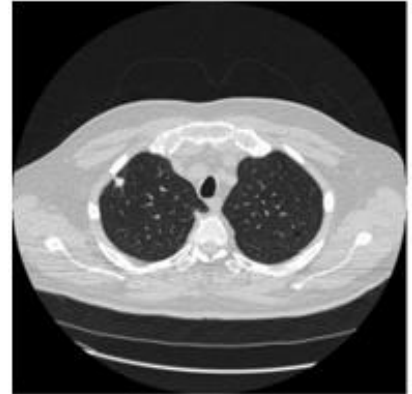

(a)

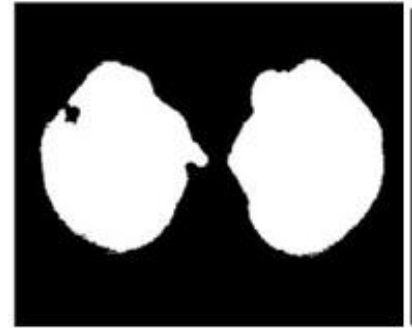

(d)

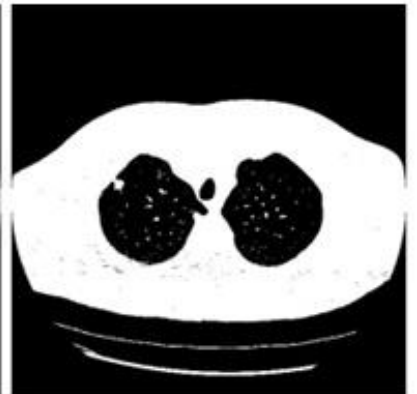

(b)

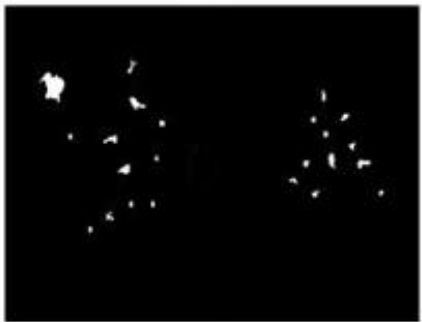

(e)

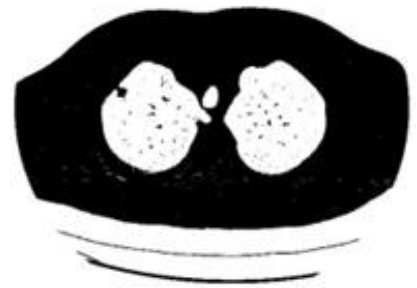

(c)

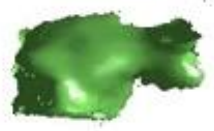

(f)

Figure 2. (a) Original CT 2-D slice, (b) cluster-2 seed mask, (c) cluster-1 seed mask, (d) Lung mask with juxta nodule hole, (e) Segmented nodule candidates, (f) 3-D visualization of one segmented nodule.

\section{Nodule Feature Extraction}

Most of the segmented nodule candidates are not malignant. To classify the malignant and benign nodules, different shape, and texture based features are extracted on the segmented nodule candidates ${ }^{20,21}$. The quantitative features must give a discriminative value for malignant and benign nodules. In this work shape and texture features are computed in both 2D and 3D planes of nodule candidates. All the shape features are extracted from the binary version of nodules and the texture features are obtained from the original DICOM version of the nodule portion.

The probability of lung nodule being benign increases with the regularity in its shape across consecutive CT slices. The more regular the shape of the nodule, the more likely it is benign. In this work, the variation in shape features in the consecutive slices are quantitatively modeled through the features such as a change in centroid, change in area and change in equivalent diameter of the nodule. Edge sharpness is also one another measure which helps to discriminate malignant nodules from benign as the benign nodules have smooth edges. Frequency domain feature of DC constant is also computed in this work, which is used to identify the calcifications. As the calcifications are uniform throughout its region, the DC constant value of it is on the higher side, and also the calcifications have a higher value of homogeneity and autocorrelation due to its uniform surface nature. The expressions for the features used in this work are tabulated in Table 1. 
Table 1: List of features extracted on a nodule

\begin{tabular}{|c|c|c|}
\hline Area & 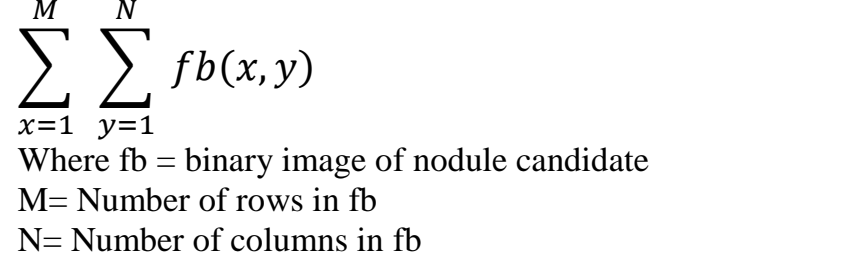 & (1) \\
\hline Diameter & $\sqrt{\frac{4 * \text { Area }}{\pi}}$ & 2) \\
\hline Centroid & {$\left[\frac{\sum_{i=1}^{M} \sum_{j=1}^{N} i * f(i, j)}{\sum_{i=1}^{M} \sum_{j=1}^{N} f(i, j)}, \frac{\sum_{i=1}^{M} \sum_{j=1}^{N} j * f b(i, j)}{\sum_{i=1}^{M} \sum_{j=1}^{N} f b(i, j)}\right.$} & (3) \\
\hline Elongation & $\frac{\max \left(x_{\text {length }}, y_{\text {length }}, z_{\text {length }}\right)}{\min \left(x_{\text {length }}, y_{\text {length }}, z_{\text {length }}\right)}$ & (4) \\
\hline Volume & $\sum_{x=1}^{m} \sum_{y=1}^{n} \sum_{z=1}^{p} f b(x, y, z)$ & $(5)$ \\
\hline Edge Sharpness & $\frac{\text { Magnitude }(\text { gradient }(\mathrm{f}(\mathrm{i}, \mathrm{j}))}{\text { length }\left(x_{\text {gradient }}\right)}$ & (6) \\
\hline Compactness & $\frac{\text { the radius of a sphere with equivalent radius }}{\text { root mean square distance from a center }}$ & (7) \\
\hline $\begin{array}{l}\text { Bounding box } \\
\text { dimension rate }\end{array}$ & $\frac{\min ([\text { [Length, yLength, zLength }])}{\max ([\text { xLength, yLength, zLength }])}$ & (8) \\
\hline Compactness2 & $\frac{\text { Surface Area }}{\text { Volume }^{2} * 36 * \pi}$ & (9) \\
\hline $\begin{array}{l}\mathrm{x} \text {-y plane projection } \\
\text { compactness }\end{array}$ & $\frac{4 * \pi * A}{P^{2}}$ & (10) \\
\hline Homogeneity & $\sum_{i=1}^{m} \sum_{j=1}^{n} \frac{P[i, j]}{1+|i-j|}$ & (11) \\
\hline Correlation & $\sum_{i=1}^{m} \sum_{j=1}^{n} \frac{(i * j) * P[i, j]-(\mu x * \mu y)}{(\sigma x * \sigma y)}$ & (12) \\
\hline mean contrast & $\frac{\text { Mean Inside }- \text { Mean outside }}{\text { Mean Inside }+ \text { Mean outside }}$ & (13) \\
\hline
\end{tabular}




\begin{tabular}{|c|c|c|}
\hline moment 6 & $\frac{\sum_{x=1}^{m} \sum_{y=1}^{n} y^{6} * f(x, y)}{\sum_{x=1}^{m} \sum_{y=1}^{n} f(x, y)}$ & (14) \\
\hline moment 7 & $\frac{\sum_{x=1}^{m} \sum_{y=1}^{n} y^{7} * f(x, y)}{\sum_{x=1}^{m} \sum_{y=1}^{n} f(x, y)}$ & $(15)$ \\
\hline 3D variance & $\begin{array}{l}\cdot\left[\frac{1}{n}\left(\sum_{i=1}^{n}\left(x i-x \_ \text {mean }\right)^{2} \sum_{y=1}^{n}\left(y i-y_{-} \text {mean }\right)^{2} \sum_{z=1}^{n}(z i-\right.\right. \\
\left.\left.\left.z_{-} \text {mean }\right)^{2}\right)\right]^{\frac{1}{2}}\end{array}$ & $(16)$ \\
\hline 3D Skewness & $\frac{E\left[\left(x-x_{\text {mean }}\right),\left(y-y_{\text {mean }}\right)\left(z-z_{\text {mean }}\right)\right]^{3}}{3 D_{-} \text {variance }}$ & $(17)$ \\
\hline Fourier DC constant & $\begin{array}{l}\mathrm{fc}(\mathrm{x}, \mathrm{y})=(-1)(\mathrm{x}+\mathrm{y}) * \mathrm{f}(\mathrm{x}, \mathrm{y}) \\
\mathrm{F}(\mathrm{u}, \mathrm{v})=\sum_{\mathrm{x}=0}^{\mathrm{M}-1} \sum_{\mathrm{y}=0}^{\mathrm{N}-1} \mathrm{fc}(\mathrm{x}, \mathrm{y}) * \exp \left[-\mathrm{j} 2 \Pi\left(\frac{\mathrm{mu}}{\mathrm{M}}+\frac{\mathrm{nv}}{\mathrm{N}}\right)\right] \\
\text { DC constant }=\mathrm{F}(\mathrm{M} / 2, \mathrm{~N} / 2)\end{array}$ & $(18)$ \\
\hline
\end{tabular}

\section{Two-Stage Hybrid Classifier}

In this section, a two-stage hybrid classifier is developed to classify the malignant and benign nodules using the selective features extracted in the previous section. The rule-based scheme followed by ANN based classifier is utilized in this chapter to classify the lung nodules.

The first stage rule-based classifier is framed with the following rules:

Table 2: Rules of the first stage classifier

\begin{tabular}{|c|c|}
\hline Nodule diameter & dia_min to dia_max (3mm to $30 \mathrm{~mm})$ \\
\hline Nodule Area & $\pi *\left(\frac{d i a \_m i n}{2}\right)^{2}$ to $\pi *\left(\frac{d i a \_\max }{2}\right)^{2}$ \\
\hline Nodule Volume & $3 * \frac{\pi}{4} *\left(\frac{\text { dia_min }}{2}\right)^{3}$ to $3 * \frac{\pi}{4} *\left(\frac{\text { dia_max }}{2}\right)^{3}$ \\
\hline Nodule Elongation & $\frac{\max \left(x_{\text {length }}, y_{\text {length }}, z_{\text {length }}\right)}{\min \left(x_{\text {length }}, y_{\text {length }}, z_{\text {length }}\right)}<4 \mathrm{~mm}$ \\
\hline Nodule round degree & $\frac{4 * \pi * \text { area }^{\text {perimeter }}{ }^{2}}{>(1 / 6) \mathrm{mm}}$ \\
\hline Nodule Centroid change & Centroid change in consecutive three slices $<10$ pixels \\
\hline
\end{tabular}

After performing the first stage rule-based classifier, most of the vessel structures, a line like irrelevant structures are removed from nodule candidate list. The nodule elongation criteria as $4 \mathrm{~mm}$ and round degree criteria as (1/6) $\mathrm{mm}$ are fixed after applying and analyzing these rules on a training image database considered for this work. All the vessels like structures and highly nonlinear irrelevant tissue clusters are removed successfully from nodule candidates 
group, but still more non-nodule and benign tissue clusters exist in the output of the first stage of classifier.

All the shape and texture features are calculated on the nodule candidates remained after the first stage of classifier. As the radiologist report on training dataset is available, the correlation analysis is performed for each feature variable against the target (malignant or benign). The correlation coefficient between each feature and the target is computed. From the correlation matrix, the features which have high correlation coefficient are picked as input features to the ANN classifier. Finally, a hybrid input feature vector is formed with twelve features (diameter, edge sharpness, circularity, compactness, bounding box dimension rate, compactness 2 , $\mathrm{x}-\mathrm{y}$ plane projection compactness, mean contrast, moment 6 , moment $7,3 \mathrm{D}$ variance and 3D skewness).

\section{Lung Nodule Growth Measurement and Prediction}

One major factor that the physicians are using in their qualitative analysis to make a call on malignant nodule is its growth. The doctors instruct the patient to undergo one another CT scan after a time span of 3 to 18 months if they suspect any symptoms in their earlier CT scan. Then by comparing the two CT scans, based on the growth of the suspected nodules, they may take a final call of its malignancy and instruct the patient to go for a biopsy. In this work, we quantitatively measure the nodules growth which helps the physicians to diagnose. The growth of the lung nodule must be measured regarding its volume than area. Any small change in the 2D area of the nodule may result in a huge change in the 3D volume. The volumes of real final nodules are computed from both the scan of each patient taken at different time intervals. The Rate of Nodule Growth (RNG) is calculated using the equation 19

$\mathrm{RNG}=\frac{\mathrm{V} 2-\mathrm{V} 1}{\mathrm{~V} 1}$

where v1 and $\mathrm{v} 2$ are the volumes of nodules segmented from earlier and follow-up scan respectively.

A total of 34 real nodules were segmented from VOLCANO and RNG was computed which is given in Table 3.Three cases showed an increase in the volume of more than double. The corresponding RNG value for case no 1, 3 and 44 was $14.6,1.46$, and 0.96 which was found to grow approximately by $15,1.5$ and 1 folds, respectively, compared with the initial scan. This noticeable growth confirms that these nodules are malignant in nature. There were 14 cases for which the RGN ranged between 0.2 and 0.8 , suggesting a reasonable growth that requires repetition of a scan after few days to confirm malignancy. Of the remaining 17 cases, 7 had RNG of less than 0.2 , i.e., almost no change and 10 cases showed minimum nodule growth which may require follow-up scans.

Also in this work, we developed a framework to predict the nodules which have the chance to grow by analyzing the earlier CT scan. Compactness, Mass Deficit Coefficient, Mass Excess coefficient and Isotropic Factor are the four measures used in this work to quantitatively model the nodules which have the higher probability to grow in future. 
Table 3: Lung nodule growth measurement

\begin{tabular}{|c|c|c|c|c|c|}
\hline Case No & $\begin{array}{l}\text { Database } \\
\text { Case ID }\end{array}$ & $\begin{array}{l}\text { Volume } \\
\text { Scan1( pixels) }\end{array}$ & $\begin{array}{l}\text { Volume } \\
\text { Scan2(pixels) }\end{array}$ & $\begin{array}{l}\text { Volume } \\
\text { Diff }\end{array}$ & RNG \\
\hline 1 & 1 & 630 & 9840 & 9210 & 14.619 \\
\hline 2 & 2 & 2108 & 2576 & 468 & 0.222 \\
\hline 3 & 6 & 95 & 234 & 139 & 1.4632 \\
\hline 4 & 7 & 149 & 181 & 32 & 0.2148 \\
\hline 5 & 8 & 90 & 91 & 1 & 0.0111 \\
\hline 6 & 10 & 1490 & 1508 & 18 & 0.0121 \\
\hline 7 & 11 & 5640 & 5970 & 330 & 0.0585 \\
\hline 8 & 13 & 8197 & 8746 & 549 & 0.067 \\
\hline 9 & 15 & 447 & 646 & 199 & 0.4452 \\
\hline 10 & 16 & 284 & 318 & 34 & 0.1197 \\
\hline 11 & 18 & 3709 & 3839 & 130 & 0.035 \\
\hline 12 & 20 & 1340 & 1353 & 13 & 0.0097 \\
\hline 13 & 21 & 2239 & 2233 & 6 & 0.0027 \\
\hline 14 & 23 & 2807 & 3031 & 224 & 0.0798 \\
\hline 15 & 24 & 930 & 901 & 29 & 0.0322 \\
\hline 16 & 25 & 1485 & 1961 & 476 & 0.3205 \\
\hline 17 & 28 & 956 & 1340 & 384 & 0.4017 \\
\hline 18 & 29 & 64 & 66 & 2 & 0.0313 \\
\hline 19 & 32 & 112 & 124 & 12 & 0.1071 \\
\hline 20 & 33 & 643 & 649 & 6 & 0.0093 \\
\hline 21 & 34 & 106 & 160 & 54 & 0.5094 \\
\hline 22 & 36 & 1028 & 1098 & 70 & 0.0681 \\
\hline 23 & 37 & 1818 & 2430 & 612 & 0.3366 \\
\hline 24 & 38 & 1742 & 1835 & 93 & 0.0534 \\
\hline 25 & 39 & 1920 & 2533 & 613 & 0.3193 \\
\hline 26 & 40 & 773 & 954 & 181 & 0.2342 \\
\hline 27 & 41 & 1236 & 1566 & 330 & 0.267 \\
\hline 28 & 42 & 611 & 850 & 239 & 0.3912 \\
\hline 29 & 44 & 690 & 1356 & 666 & 0.9652 \\
\hline 30 & 45 & 866 & 1223 & 357 & 0.4122 \\
\hline 31 & 46 & 136 & 149 & 13 & 0.0956 \\
\hline 32 & 47 & 1688 & 2111 & 423 & 0.2506 \\
\hline 33 & 48 & 653 & 381 & 272 & 0.7139 \\
\hline 34 & 49 & 4280 & 4950 & 670 & 0.1565 \\
\hline
\end{tabular}


Table 4: Nodule growth predictor features

Mass Deficit (MD)

Isotropic Factor(IF)
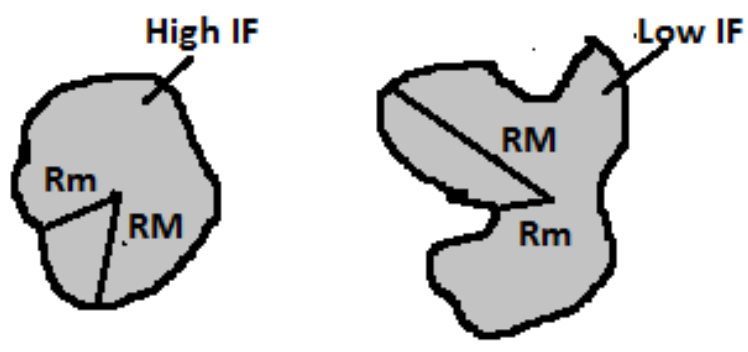

$\mathrm{IF}=\frac{\mathrm{Rm}}{\mathrm{RM}}$

\section{RESULTS AND DISCUSSION}

In this work 24 patient lung CT scan series from VOLCANO database and five patient cases from LIDC database are used as a training image set. VOLCANO cases contain 25 to $\mathrm{CT}$ image slices per scan as an average, and LIDC scans have 120 to $280 \mathrm{CT}$ slices per scan. In this work, a total of $1450 \mathrm{CT}$ cross-sectional images (slices) is used for training.

The auto-seed clustering based segmentation discussed before was effectively applied on all these $1450 \mathrm{CT}$ slice images to segment all the possible nodule candidates. For juxta-nodule, the morphological closing is performed to close the juxta-pleural hole present in the parenchyma region after applying the segmentation process. The juxta-pleural nodule segmentation case is shown in figure 3 .

The nodule candidates segmented from these 1450 CT slices are applied to the rule-based classifier (first stage classifier). The nodule candidates, which are not satisfying the rules mentioned in Table 2 are eliminated. The training database 
set used in this work contains 32 malignant nodules. The first stage of classifier output preserving all the 32 nodules, which marked in radiologist report. Along with these 32 nodules, 362 other nodules also remained in the output of the rulebased classifier. Hence, the True Positive (TP) (the nodules detected which matched with radiologist report) at the output of classifier first stage is 32, and the False Positive (FP) (the nodules detected, which not matched with radiologist report) is 362. As all the nodules marked by the radiologists report are detected, the False Negative (FN) is zero. The performance of the first stage rule-based classifier is shown in Table 5.

Table 5. Performance measure of the first stage of classifier

\begin{tabular}{ccc}
\hline Statistic & Formula & Value \\
\hline Sensitivity & $\frac{\mathrm{TP}}{\mathrm{TP}+\mathrm{FN}}$ & $100 \%$ \\
\hline False Positive & FP/scan & 12.48 \\
\hline
\end{tabular}

Artificial Neural Network based the second stage of classifier is used to reduce the false positive. The input feature matrix size for an ANN is $12 \times 394$, where 12 is the total number of finalized features and 394(362+32) is the number of nodule candidates remained at the output of the rule-based classifier. The target matrix of size $2 \times 394$ is framed with the help of radiologist findings. The target output of [ $\left[\begin{array}{ll}0 & 1\end{array}\right]$ and [ 10$]$ is fixed for benign and malignant nodules respectively. The BPN based ANN classifier is trained towards the minimum error. The trained net input and output weights are saved for testing new datasets.

10 cases of VOLCANO and 5 cases of LIDC are tested using the parameters of trained classifier. This testing set contains 18 malignant nodules. Total of 975 cross-sectional CT images are present in the testing database. After the segmentation process, all the suspected nodule candidates are applied under the rule based classifier. Total of 204 nodule candidates remained at the output of the rule-based classifier. The 12 features discussed previously are computed for each nodule and the feature vector of size $12 \times 283$ is applied to a trained ANN system. The benign nodules feature vector are converged very near to [0 1], and the malignant nodules are to [1 0$]$. The output of our designed lung nodule detection system is compared with the radiologist report. Algorithm developed in this work correctly detected 16 malignant nodules out of 18 and missed to detect 2, with a TP of 16 and FN of 2. This algorithm successfully eliminated 170 nodule candidates and wrongly identified 34 nodule candidates as a malignant nodule. Therefore, the TN is 170; FP is 34 ; and the FP per patient scan is 2.26. The overall performance measure of the developed algorithm is given in Table 6.

The nodules from VOLCANO database are analyzed further for its growth measurement and prediction, as this database have two CT scan series for each patient which taken at different time intervals. In this work, the nodule growth is predicted using four measures: compactness (CO), mass excess (ME), the mass deficit (MD) and isotropic factor (IF). These values are computed for 34 patient cases. The nodules which had larger growth having $\mathrm{CO}<0.4$, IF $<0.4$, $\mathrm{MD}>0.3$ and $\mathrm{ME}>0.6$. The nodules which had average growth having $\mathrm{CO}$ in 
between 0.4 to 0.6 , IF in between 0.5 to 0.75 , MD in between 0.2 to 0.4 and $\mathrm{ME}$ in between 0.2 to 0.5 . Finally, the nodules which had very minimum or no growth have the $\mathrm{CO}>0.6, \mathrm{IF}>0.7, \mathrm{MD}$ and $\mathrm{ME}<0.2$. Therefore, the nodules which have low $\mathrm{CO}$, low IF, high MD and high ME values might have the potential to grow.

Table 6. Statistical results

\begin{tabular}{ccc}
\hline Statistic & Formula & Value \\
\hline Sensitivity & $\frac{T P}{T P+F N}$ & $88.8 \%$ \\
\hline Specificity & $\frac{T N}{T N+F P}$ & $83.3 \%$ \\
\hline FP/patient scan & Total FP / Number of patient scan & 2.26 \\
\hline Disease prevalence & $\frac{T P+F N}{T P+F N+T N+F P}$ & $8.1 \%$ \\
\hline
\end{tabular}

\section{CONCLUSION}

An efficient and automatic lung nodule detection system was successfully implemented in this work. Auto-seed clustering based segmentation was carried out in this work to segment all possible nodule candidates. Block histogram processing technique was used in this study to initialize and update the cluster seed value automatically. After analyzing many shape and texture features, six features were used to form the rules for the first stage of classifier. Other twelve features were computed on the nodules passing through the first stage of classifier. The BPN-ANN network was used as the second stage of the classifier to classify the malignant and benign nodules. This algorithm proposed in this paper produced a false positive of 2.26 per patient scan with a reasonable sensitivity of $88.8 \%$. The nodules growing nature was modeled in this work using four shape measures. In future, this work can be extended by modeling the nodule growth prediction algorithm, more accurately by training and testing with more patient cases.

\section{REFERENCES}

1. Torre, L.A., Bray, F., Siegel, R.L., Ferlay, J., Lortet Tieulent, J. and Jemal, A., 2015. Global cancer statistics, 2012. CA: CA Cancer J Clin, 65(2), pp.87-108.

2. Chheang, S. and Brown, K., 2013, June. Lung cancer staging: clinical and radiologic perspectives. Semin Intervent Rad, Thieme Medical Publishers, 30(2),pp. 099-113.

3. Armato, S.G. and Sensakovic, W.F., 2004. Automated lung segmentation for thoracic CT: impact on computer-aided diagnosis1. Acad Radiol, 11(9), pp.1011-1021.

4. Leader, J.K., Zheng, B., Rogers, R.M., Sciurba, F.C., Perez, A., Chapman, B.E., Patel, S., Fuhrman, C.R. and Gur, D., 2003. Automated lung segmentation in X-ray computed tomography: development and evaluation of a heuristic threshold-based scheme1. Acad Radiol, 10(11), pp.1224-1236.

5. Dehmeshki, J., Amin, H., Valdivieso, M. and Ye, X., 2008. Segmentation of pulmonary nodules in thoracic CT scans: a region growing approach. IEEE T Med Imaging, 27(4), pp.467-480 
6. Mehtre, B.M., Kankanhalli, M.S. and Lee, W.F., 1997. Shape measures for content based image retrieval: a comparison. Inform Process Manag, 33(3), pp.319-337

7. Han, F., Wang, H., Zhang, G., Han, H., Song, B., Li, L., Moore, W., Lu, H., Zhao, H. and Liang, Z., 2015. Texture feature analysis for computer-aided diagnosis on pulmonary nodules. J Digit Imaging, 28(1), pp.99-115.

8. Choi, W.J. and Choi, T.S., 2014. Automated pulmonary nodule detection based on threedimensional shape-based feature descriptor. Comput Meth Prog Bio, 113(1), pp.37-54.

9. Shen, W., Zhou, M., Yang, F., Yang, C. and Tian, J., 2015, June. Multi-scale convolutional neural networks for lung nodule classification. In International Conference on Information Processing in Medical Imaging (pp. 588-599). Springer International Publishing.

10. Chan, H.P., Sahiner, B., Hadjiyski, L., Zhou, C. and Petrick, N., The Regents Of The University Of Michigan Technology Management Office, 2003. Lung nodule detection and classification. U.S. Patent Application 10/504,197.

11. Kostis, W.J., Reeves, A.P., Yankelevitz, D.F. and Henschke, C.I., 2003. Three-dimensional segmentation and growth-rate estimation of small pulmonary nodules in helical CT images. IEEE T Med Imaging, 22(10), pp.1259-1274.

12. Zheng, Y., Steiner, K., Bauer, T., Yu, J., Shen, D. and Kambhamettu, C., 2007, October. Lung nodule growth analysis from 3D CT data with a coupled segmentation and registration framework. In 2007 IEEE 11th International Conference on Computer Vision (pp. 1-8). IEEE.

13. Hasegawa, M., Sone, S., Takashima, S., Li, F., Yang, Z.G., Maruyama, Y. and Watanabe, T., 2000. Growth rate of small lung cancers detected on mass CT screening. Brit J Radiol, 73(876), pp.1252-1259.

14. Armato III, S.G., McLennan, G., Bidaut, L., McNitt-Gray, M.F., Meyer, C.R., Reeves, A.P., Zhao, B., Aberle, D.R., Henschke, C.I., Hoffman, E.A. and Kazerooni, E.A., 2011. The lung image database consortium (LIDC) and image database resource initiative (IDRI): a completed reference database of lung nodules on CT scans. Med Phys, 38(2), pp.915-931.

15. Reeves, A.P., Jirapatnakul, A.C., Biancardi, A.M., Apanasovich, T.V., Schaefer, C., Bowden, J.J., Kietzmann, M., Korn, R., Dillmann, M., Li, Q. and Wang, J., 2009, September. The VOLCANO'09 challenge: Preliminary results. In Second international workshop of pulmonary image analysis (pp. 353-364).

16. Kumar, T.S. and Ganesh, E.N., 2013. Proposed technique for accurate detection/segmentation of lung nodules using spline wavelet techniques. Int. J. Biomed. Sci, 9(1), pp.9-17.

17. Krishnamurthy, S., Narasimhan, G. and Rengasamy, U., 2016. Three-dimensional lung nodule segmentation and shape variance analysis to detect lung cancer with reduced false positives. P I Mech Eng H, 230(1), pp.58-70.

18. SenthilKumar Krishnamurthy, Ganesh Narasimhan, Umamaheswari Rengasamy. (in press) "Lung nodule growth measurement and prediction using auto cluster seed k-means morphological segmentation and shape variance analysis", Int J Biomed Eng Technol.

19. Reed, T.R. and Dubuf, J.H., 1993. A review of recent texture segmentation and feature extraction techniques. CVGIP: Image understanding, 57(3), pp.359-372.

20. Zhang, D. and Lu, G., 2004. Review of shape representation and description techniques. Pattern recognition, 37(1), pp.1-19.

21. Mukundan, R. and Ramakrishnan, K.R., Moment functions in image analysis: theory and applications (Vol. 100). Singapore: World Scientific, 1998. 